\title{
Globe
}

Revue internationale d'études québécoises

\section{Leigh Oakes et Jane Warren : Language, Citizenship and Identity in Quebec, Houndmills/Basingstoke/Hampshire/New York, Palgrave Macmillan, 2007}

\section{Wim Remysen}

Volume 11, numéro 2, 2008

URI : https://id.erudit.org/iderudit/1000531ar

DOI : https://doi.org/10.7202/1000531ar

Aller au sommaire du numéro

Éditeur(s)

Globe, Revue internationale d'études québécoises

ISSN

1481-5869 (imprimé)

1923-8231 (numérique)

Découvrir la revue

Citer ce compte rendu

Remysen, W. (2008). Compte rendu de [Leigh Oakes et Jane Warren : Language, Citizenship and Identity in Quebec, Houndmills/Basingstoke/Hampshire/New

York, Palgrave Macmillan, 2007]. Globe, 11(2), 213-219.

https://doi.org/10.7202/1000531ar d'utilisation que vous pouvez consulter en ligne.

https://apropos.erudit.org/fr/usagers/politique-dutilisation/ 


\section{Leigh Oakes et Jane Warren}

Language, Citizenship and Identity in Quebec

Houndmills/Basingstoke/Hampshire/New York, Palgrave Macmillan, 2007.

Publié dans la collection "Language and Globalization " et préfacé par Gérard Bouchard, l'ouvrage Language, Citizenship and Identity in Quebec s'interroge sur les rapports complexes qui existent entre la langue française et l'identité nationale au Québec. Les aureurs de l'ouvrage cherchent plus particulièrement à comprendre comment le Québec cherche à s'affirmer en tant que société francophone à l'échelle tant internationale que locale, c'est-à-dire en tenant compte des enjeux soulevés tant par la mondialisation que par la diversité ethnique dans la province même. À ce propos, les auteurs considèrent que le rapport déposé en 2001 par la Commission des États généraux sur la situation et l'avenir de la langue française au Québec ${ }^{11}$ - mieux connue sous le nom de Commission Larose - constitue un point tournant dans la réflexion sur la place que la langue occupe dans la construction de l'identicé nationale des Québécois. Selon eux, ce rapport illustre bien que le Québec tente dorénavant d'adopter une approche civique en matière de politique linguistique, dans le but de réunir l'ensemble des Québécois peu importe leur origine ethnique.

L'introduction fait état des objectifs, de la méthodologie et du cadre théorique. Le principal objectif de l'ouvrage consiste à alimenter le débat actuel à propos de l'orientation civique que le Québec entend donner à sa politique linguistique, mais dans une perspective résolument sociolinguistique. En d'autres termes, les auteurs replacent la langue au cœur de leurs préoccupations, et ce, d'un point de vue aussi bien macrosociolinguistique (politique linguistique, attitudes linguistiques) que microsociolinguistique (variation linguistique, standardisation). Cela dit, ils adoptent aussi une approche pluridisciplinaire et ne laissent donc pas pour compre les acquis d'autres disciplines qui s'intéressent à la question de l'identité nationale, telles les sciences politiques, la philosophie politique ou la sociologie. En outre, si les auteurs considèrent que le Québec constitue un cas unique pour alimenter la réflexion sur les rapports entre langue et identité, leur approche se veut également comparative. À leur avis, une telle approche permettrait aux Québécois de pousser plus loin la réflexion sur leur propre situation, mais

$$
++\leftarrow
$$

11. GOUVERNEMENT DU QUÉBEC, Le français, une Langue pour tout le monde. Une nouvelle approche stratégrque et citoyenne, Québec, Gouvernement du Québec, 2001. 
aussi d'envisager la question de la langue et de l'identité dans une perspective plus générale.

En ce qui concerne la méthodologie, l'étude se base principalement sur l'analyse de sources primaires (publications officielles et études empiriques) et secondaires (travaux universitaires et articles de journaux). L'analyse de ces sources est complétée par une série d'entrevues semi-dirigées avec certains membres des différentes instances gouvernementales du Québec et avec des universitaires. Pour ce qui est du cadre théorique, les auteurs définissent les principaux concepts qui ont orienté leur étude: identité sociale, ethnique et nationale, et mondialisation. Dans la mesure où ces concepts donnent souvent lieu à des interprétations différentes, leur but consiste à préciser comment ils entendent s'en servir plutôt que de faire une analyse critique de l'ensemble des positions théoriques auxquelles ils ont pu donner lieu.

L'ouvrage est structuré en trois parties qui correspondent à autant de questions de recherche qui ont orienté l'étude: 1) la construction d'une identité nationale québécoise dans le contexte de la mondialisation et de la diversité ethnique au Québec; 2) la promotion du français comme langue publique commune pour l'ensemble des Québécois; 3) l'expérience vécue par les minorités au Québec relativement à la place du français dans la province.

Dans la première partie ("New Challenges"), les auteurs analysent comment le Québec prend des mesures concrètes pour mieux faire face aux défis soulevés par la diversité ethnique de sa population et par la mondialisation, dans le but de promouvoir le français comme facteur de cohésion sociale.

Le deuxième chapitre ("From French Canadian to Quebecer") examine comment le Québec en est venu à adopter un modèle de nation civique - plutôt qu'ethnique - depuis les modifications importantes que la société québécoise a subies à partir de la Révolution tranquille. Selon les auteurs, la modernisation et la sécularisation du Québec sont à l'origine de la "territorialisation" de l'identité québécoise, ce qui veut dire que les Québécois se définissent désormais comme une majorité francophone dans leur province plutôt que comme une minorité au sein du Canada. Le chapitre traite aussi de la possibilité de créer une citoyenneté québécoise à côté de la citoyenneté canadienne. De l'avis des auteurs, cette question dépend largement de la conception qu'on peut se faire de la notion de "citoyenneté", notamment lorsqu'on la situe par rapport à celle de "nationalité». C'est pourquoi il est possible, selon eux, de mener le débat sur la citoyenneté 
québécoise sans l'associer directement au débat sur la souveraineté du Québec, comme on a souvent tendance à le faire.

D'orientation plus théorique, le troisième chapitre («Redefining the Quebec Nation") présente une vue d'ensemble des différents modèles de nation qui ont été proposés pour le Québec: le modèle ethnique (Dumont), le modèle civique (Derriennic, Leydet, Caldwell et Bariteau) et le modèle intégrationniste, à la fois ethnique et civique (Bouchard, Seymour, Taylor). Dans ce chapitre, les auteurs ne se contentent pas de présenter et d'évaluer ces trois modèles: ils prennent clairement position dans le débat. Ainsi, ils sont d'avis que le Québec a tout intérêt à promouvoir un modèle de société intégrationniste, c'est-à-dire un modèle qui jette les bases d'une société civique et inclusive, mais qui reconnaît en même temps l'importance de l'identité ethnique du groupe francophone majoritaire.

Le quatrième chapitre ("Quebec in a Globalising World») s'intéresse à la voix que le Québec cherche à se donner sur la scène internationale dans le but de consolider sa propre identité linguistique et culturelle. À ce sujet, les auteurs estiment que le Québec réussit à tirer avantage des défis soulevés par la mondialisation : tout en plaidant, sur le plan international, pour la protection de la diversité linguistique et culturelle, il parvient à promouvoir et à consolider sa propre identité locale. Les auteurs présentent plus particulièrement comment le Québec cherche à promouvoir le statut du français dans les Amériques à côté des autres langues principalement en usage sur les continents nord- et sud-américains (l'anglais, l'espagnol et le portugais) et comment il se profile au sein de la Francophonie comme défenseur de la diversité culturelle.

Dans la deuxième partie de l'ouvrage ("A Common Language»), les auteurs se penchent sur les interventions politiques en matière de langue dans la province au cours des dernières décennies, qu'il s'agisse d'actions sur le statut (chapitre 5) ou sur le corpus (chapitre 6).

La protection et la promotion du statut du français font l'objet du cinquième chapitre ("French: A Language for All Quebecers"). Dans cette section, les auteurs observent comment le Québec a progressivement dissocié le français de l'ethnicité dans le but d'encourager les immigrants à adopter l'usage de cette langue et, partant, d'en assurer la survie. Cette nouvelle orientation passe par la promotion du français comme "langue publique commune", c'est-à-dire comme dénominateur commun pour former une identité nationale pour l'ensemble des Québécois, peu importe leur origine. Comme dans le troisième chapitre, les auteurs sont toutefois d'avis qu'il est impossible de complètement "désethniciser" la langue. En d'autres termes, 
toute politique linguistique au Québec doit selon eux tenir compte du fait que le français constitue une composante importante de l'identité ethnique du groupe majoritaire.

Le sixième chapitre ("Whose French? Language Attitudes, Linguistic Insecurity and Standardisation") aborde la question de la qualité de la langue. Après avoir retracé les origines de l'insécurité linguistique des Québécois, les auteurs se demandent quelle variété de français le Québec devrait promouvoir: un français international ou un français québécois? À ce propos, ils considèrent que le concept de "français international" tel que défendu par certains linguistes relève du mythe plutôt que de la réalité. Ils sont pour leur part convaincus qu'une vision pluricentrique du français vision qui admet l'existence de plusieurs variétés nationales de français, dont une variété québécoise, dotées d'un registre standard qui leur est propre - est non seulement plus proche de la réalité des usages linguistiques, mais aussi plus apte à faire du français un symbole important de l'identité nationale de tous les Québécois. Les auteurs passent également en revue les différents défis que soulève la description de cette variété québécoise, notamment dans le cadre d'un dictionnaire.

La dernière partie de l'ouvrage ("Diverse Experiences") est consacrée aux rapports que les trois principales communautés minoritaires présentes au Québec (les immigrants, les anglophones et les autochtones) entretiennent avec le français et avec l'identité québécoise. Dans cette partie, les auteurs ne s'intéressent pas seulement à la place que ces minorités occupent dans le discours officiel sur la langue au Québec, mais aussi aux opinions des individus qui appartiennent à l'un ou l'autre de ces groupes.

Dans le septième chapitre ("Language, Immigration and Belonging"), les auteurs se concentrent sur l'importance que les immigrants accordent au français dans leur identité et ils analysent la place que le français occupe comme langue d'usage dans leur vie quotidienne. Selon les études consultées par les auteurs, bon nombre d'immigrants développent un sentiment d'appartenance au Québec et un attachement au français. Par contre, ces immigrants développent souvent un attachement tout particulier pour la culture montréalaise (plutôt que pour la culture québécoise dans son ensemble) et ils accordent une importance particulière au bilinguisme françaisanglais (plutôt qu'au seul français), ce qui pose tout un défi pour les autorités du Québec qui cherchent leur adhésion au français.

La place de la communauté anglophone au Québec est abordée dans le huitième chapitre ("Transformations of Anglophone Quebec»). Les auteurs soulignent la difficulté de bien définir cette communauté compte 
tenu de ses diverses origines; par exemple, il n'est pas toujours clair si cette communauté doit inclure les immigrants qui ont adopté l'anglais mais qui continuent de se servir de leur langue maternelle à la maison. En outre, la perception souvent négative des francophones à l'égard de cette communauté explique, selon les auteurs, que les anglophones ne soient pas toujours considérés comme des Québécois à part entière par la majorité francophone, même si les deux communautés se rapprochent davantage.

Le neuvième chapitre aborde la question des droits linguistiques des différentes communautés autochtones au Québec ("Linguistic Rights for Aboriginal Nations»). Étant donné que ces communautés sont elles-mêmes préoccupées par la défense et la survie de leur propre identité linguistique et culturelle, elles adoptent souvent une attitude négative à l'endroit du français (voire à l'endroit de l'anglais), qu'elles jugent menaçant. Au dire des auteurs, la politique linguistique actuelle au Québec permet difficilement de concilier la promotion du français avec les préoccupations identitaires auxquelles les autochtones doivent faire face, ce qui n'est pas sans soulever des problèmes dans la promotion du français au sein de ces communautés.

Dans la conclusion de l'ouvrage, les auteurs soulignent que le Québec a tout intérêt à poursuivre sur la voie dans laquelle il s'est engagé au cours des dernières décennies, s'il souhaite continuer à promouvoir une société francophone inclusive, ouverte à tous les habitants de la province. Ils insistent néanmoins sur trois aspects qui méritent, à leur avis, une attention toute particulière de la part des autorités pour que la politique linguistique québécoise soit fructueuse. D'abord, le modèle civique tel que mis de l'avant par le Québec ne réussit pas à rallier tous les immigrants, pour qui le français reste d'abord et avant tout un simple instrument de communication, pour lequel ils ne développent aucun attachement plus profond. Ensuite, le Québec doit continuer à promouvoir et à décrire une variété de français qui sera partagée par l'ensemble des membres de la société québécoise, toutes origines ethniques confondues, mais qui soit apte à exprimer l'identité ethnique et culturelle du groupe majoritaire. Enfin, il importe que le Québec reconnaisse l'importance de l'identité ethnique non seulement du groupe francophone majoritaire, mais aussi des groupes minoritaires. En d'autres mots, la place du français au Québec ne peut pas être conçue exclusivement en termes civiques.

À notre avis, les qualités de cet ouvrage sont multiples, et nous nous limiterons ici à en souligner trois. Tout d'abord, même s'il s'adresse en premier lieu à un public plus spécialisé, Language, Citizenship and Identity in Quebec est un ouvrage accessible qui sera lu avec intérêt par quiconque 
s'intéresse à la question de l'identité québécoise et de la place du français au Québec. Ensuite, s'il est vrai que les auteurs s'interrogent principalement sur l'approche civique acruellement adoptée par le Québec, ils accordent aussi, dans plusieurs chapitres, une place importante au contexte historique plus large. Grâce à cette perspective, le lecteur est davantage en mesure de suivre l'évolution du discours sur la langue au Québec et, partant, de comprendre les enjeux actuels. Enfin, comme ils le soulignent eux-mêmes (p. 3), les auteurs (tous deux d'origine australienne) adoptent un regard nouveau, externe, sur un débat largement commenté et médiatisé au Québec. Étant donné leur position particulière - les auteurs sont très au fait de la société québécoise, sans pour autant y participer quotidiennement - , ils apportent un point de vue extérieur à un débat très politisé, où la rectitude politique ainsi que les enjeux partisans ou électoraux occupent parfois une place trop importante, ce qui permet d'élargir le débat. Il suffit de relire les passages où les auteurs insistent sur l'importance de ne pas exclure toute référence ethnique de la politique linguistique au Québec pour s'en rendre compte.

Par contre, certains aspects abordés dans l'ouvrage mériteraient à notre avis d'être approfondis. D'abord, si les auteurs affirment que leur analyse porte essentiellement sur des sources primaires et secondaires, il serait intéressant de donner une place plus importante à l'opinion publique, par exemple par l'utilisation de questionnaires portant sur les attitudes linguistiques des locuteurs, de façon à mieux faire ressortir les divergences et les convergences entre le discours officiel et les jugements partagés par les Québécois eux-mêmes. Cette approche, que Oakes avait lui-même utilisée dans son étude sur l'identité nationale en France et en Suède ${ }^{12}$, n'est pas totalement absente de l'ouvrage, notamment dans la dernière partie, mais elle aurait pu être développée de façon plus poussée.

Par ailleurs, nous sommes d'avis que l'approche comparative adoptée par les auteurs pourrait être exploitée davantage. Comme le précise l'introduction (p. 3), les liens établis entre la situation québécoise et d'autres contextes se limitent essentiellement à des communautés avec lesquelles les auteurs sont plus familiers. C'est ce qui explique pourquoi ils abordent la question de l'identité et de la langue en France et en Suède (analysée par Oakes dans un ouvrage antérieur ${ }^{13}$ ) ou encore aux États-Unis et en Suisse. Par contre, certaines communautés qui présentent pourtant des similitudes plus

$$
+4
$$

12. Leigh OAKES, Language and Nastonal Identty. Comparing France and Sweden, Amsterdam/Philadelphie, John Benjamins, 2001.

13. Ibid 
frappantes avec la situation québécoise - il suffit de penser à la Flandre, à l'Écosse ou à la Catalogne - sont peu ou pas mentionnées.

Tout bien considéré, Language, Citizenship and Identity in Quebec apporte des réflexions et des points de vue importants dont il convient de tenir compte dans le débat présent sur le français au Québec. Étant donné le contexte actuel - marqué par une réflexion collective sur des questions aussi chargées émotivement que délicates à traiter (l'identité québécoise, l'intégration des minorités culturelles, les accommodements raisonnables, la place du français sur l'île de Montréal) -, la publication de cet ouvrage ne pouvait tomber mieux. On peut donc espérer qu'il sera bientôt disponible en français, puisqu'il risque d'attirer l'attention de tous les Québécois qui s'intéressent à la question de la langue et de l'identité.

Wim Remysen Université Laval

\section{Jean-Philippe Warren}

Ils voulaient changer le monde.

Le militantisme marxiste-léniniste au Québec Montréal, VLB, 2007.

Jean-Philippe Warren enseigne la sociologie à l'Université Concordia (Montréal), où il offre des cours sur les mouvements sociaux. Il est aussi et surtout un auteur très prolifique, spécialisé dans l'histoire intellectuelle des idées et des valeurs. Son dernier ouvrage s'intitule Une douce anarchie. Les années 68 au Québec ${ }^{14}$ et traite du mouvement étudiant.

En 2007, Warren signait Ils voulaient changer le monde. Le militantisme marxiste-léniniste au Québec, dans lequel il aborde la fascinante odyssée de l'activisme m-l (comme on disait) dans les années 1970 jusqu'à son effondrement, au début des années 1980. L'ouvrage emprunte plus à la forme de l'essai et du récit qu'à celle, plus convenue, des études en sociologie ou en science politique sur les mouvements sociaux. Ainsi, on cherchera en vain des références aux cadres d'analyse à la mode (mobilisation des ressources, processus politique, parcours biographique) ou aux spécialistes des théories des mouvements sociaux (Touraine, Melluci, Tilly, Tarrow, della

$$
++4
$$

14. Jean-Philippe WARREN, Une douce anarchie. Les années 68 au Quebec, Montréal, Boréal, 2008. Warren a aussı dirigé le dossier a Les mouvements étudiants des années 1960 ", Bulletın d'hssotre politique, vol. 16, $n^{\circ} 2,2008$. 